\title{
A Class of Weakly Singular Iterated Integral Inequality
}

Yusong Lu, Yangyun Ou

School of Mathematics and Statistics, Hechi University, Guangxi, Yizhou, 546300, China

\begin{abstract}
In this paper, we establish a class of weakly singular integral inequality, which consists of iterated integral. Under several practical assumptions, the inequality is solved by adopting novel analysis techniques, such as: change of variable, amplification method, and inverse function. Explicit bounds for the unknown functions are given clearly.

Keywords: Integral inequality; Iterated integrals; Weakly singular integral kernel; Analysis technique; Estimation.
\end{abstract}

\section{Introduction}

In 1997 Medved [1] discuss nonlinear singular integral inequalities

$$
u(t) \leq a(t)+\int_{0}^{t}(t-s)^{\beta-1} f(s) w(u(s)) d s,
$$

and the estimates of solutions are given.

In 2011, Abdeldaim et al. [2] studied a new integral inequality of GronwallBellman-Pachpatte type

$$
u(t) \leq u_{0}+\int_{t_{0}}^{t}\left[f(s) u(s)\left[u(s)+\int_{t_{0}}^{s} h(\tau)\left[u(\tau)+\int_{t_{0}}^{\tau} g(\xi) u(\xi) d \xi\right] d \tau\right] d s .\right.
$$

In this paper, on the basis of [1-9], we discuss a nonlinear iterated integral inequality

$$
\begin{aligned}
u(t) \leq & a(t)+\int_{t_{0}}^{t}(t-s)^{\beta_{1}-1} f(s) w_{1}(u(s))[u(s) \\
& +\int_{t_{0}}^{s}(s-\tau)^{\beta_{2}-1} g(\tau) w_{2}(u(\tau))[u(\tau) \\
& \left.\left.+\int_{t_{0}}^{\tau}(\tau-\xi)^{\beta_{3}-1} h(\xi) w_{3}(u(\xi)) d \xi\right] d \tau\right] d s,
\end{aligned}
$$

for all $t \in\left[t_{0}, J\right)$. 


\section{Main result}

For convenience, we cite the following lemma and definition:

Definition 1. (see [1])

Let $\mathrm{q}>0$ be a real number and $0<\mathrm{T}<\infty$. We say that a function $w: R^{+} \rightarrow R$ satisfies a condition (q), if

$$
e^{-q t}[w(u)]^{q} \leq R(t) w\left(e^{-q t} u^{q}\right), \forall u \in R^{+}, t \in[0, T) .
$$

where $\mathrm{R}(\mathrm{t})$ is a continuous, nonnegative function.

\section{Lemma 1.( see [1])}

Let $\beta \in[0,1 / 2], 0<p<\frac{1}{1-\beta}$, then $1+p(\beta-1)>0$ and

$$
\int_{t_{0}}^{t}(t-s)^{p(\beta-1)} e^{p s} d s \leq \frac{e^{p t}}{p^{1+p(\beta-1)}} \Gamma(1+p(\beta-1)) .
$$

Lemam 2. (see [3])

Let $\alpha>1$ is a real numbers, $\mathrm{n}$ is a natural number, and $A_{1}, A_{2}, \cdots, A_{n}$ be nonnegative real numbers. Then

$$
\left(A_{1}+A_{2}+\cdots+A_{n}\right)^{\alpha} \leq n^{\alpha-1}\left(A_{1}^{\alpha}+A_{2}^{\alpha}+\cdots+A_{n}^{\alpha}\right) .
$$

\section{Theorem 1.}

Let $\beta_{i} \in[0,1 / 2](i=1,2,3), w_{1}, w_{2}, w_{3}$, satisfy the condition (4) with $q_{i}=\left(1+\beta_{i}\right) / \beta_{i}, q_{1}<q_{2}<q_{3}, q_{2} / q_{1}<2$. If $u(t)$ satisfies (3), then

$$
u(t) \leq e^{t}\left(\Omega_{1}^{-1}\left(\Omega_{4}^{-1}\left(\Omega_{5}^{-1}(\tilde{C}(t))\right)\right)\right)^{1 / q_{1}}, \forall t \in\left[t_{0}, T_{1}\right],
$$

where

$$
\begin{aligned}
& \tilde{C}(t)=\Omega_{5}\left[\Omega_{4}\left(\Omega_{1}(C(t))+\int_{t_{0}}^{t} \tilde{h}(s) d s\right)+\int_{t_{0}}^{t} \tilde{g}(s) d s\right]+\int_{t_{0}}^{t} \tilde{f}(s) d s, \\
& \Omega_{1}(z)=\int_{z 0}^{z} \frac{d s}{w_{3}(s)}, z_{0}>0, z \in\left(z_{0},+\infty\right) \\
& \Omega_{2}(z)=\int_{z_{0}}^{z} \frac{w_{3}\left(\Omega_{1}^{-1}(s)\right) d s}{w_{2}\left(\Omega_{1}^{-1}(s)\right) \Omega_{1}^{-1}(s)}, z_{0}>0, z \in\left(z_{0},+\infty\right), \\
& \Omega_{3}(z)=\int_{z_{0}}^{z} \frac{w_{2}\left(\Omega_{1}^{-1}\left(\Omega_{2}^{-1}(s)\right)\right) d s}{w_{1}\left(\Omega_{1}^{-1}\left(\Omega_{2}^{-1}(s)\right)\right)}, z_{0}>0, z \in\left(z_{0},+\infty\right), \\
& \Omega_{4}(z)=\int_{z_{0}}^{z} \frac{w_{3}\left(\Omega_{1}^{-1}(s)\right) d s}{w_{2}\left(\Omega_{1}^{-1}(s)\right)\left(\Omega_{1}^{-1}(s)\right){ }^{2-\frac{q_{2}}{q_{1}}}}, z_{0}>0, z \in\left(z_{0},+\infty\right),
\end{aligned}
$$




$$
\begin{aligned}
& \Omega_{5}(z)=\int_{z_{0}}^{z} \frac{w_{2}\left(\Omega_{1}^{-1}\left(\Omega_{2}^{-1}(s)\right)\right) d s}{w_{1}\left(\Omega_{1}^{-1}\left(\Omega_{2}^{-1}(s)\right)\right)\left(\Omega_{1}^{-1}\left(\Omega_{2}^{-1}(s)\right)\right)^{\frac{q_{2}}{q_{1}}-1}}, z_{0}>0, z \in\left(z_{0},+\infty\right), \\
& C(t)=2^{\frac{q_{3}}{q_{2}}+\frac{q_{2}}{q_{1}}-2} B(t), \\
& B(t)=2^{q_{1}-1} a^{q_{1}}(t), \\
& \tilde{f}(t)=2^{\frac{q_{2}}{q_{1}}-2} \tilde{\widetilde{f}}(t) \text {, } \\
& \tilde{g}(t)=\frac{q_{1}}{q_{2}} 2^{\frac{q_{3}}{q_{2}}+\frac{q_{2}}{q_{1}}-2} \tilde{\widetilde{g}}(t), \\
& \tilde{h}(t)=2^{\frac{q_{3}}{q_{2}}-1} \tilde{\widetilde{h}}(t), \\
& \tilde{\tilde{f}}(s)=2^{2 q_{1}-2} K_{1} f^{q_{1}}(s) R_{1}(s) e^{q_{1} s}, \\
& \widetilde{\widetilde{g}}(\tau)=\left(2^{2 q_{2}-1} K_{2}\right)^{q_{2} / q_{1}} g^{q_{2}}(\tau) R_{2}(\tau) e^{q_{2} \tau} \text {, } \\
& \widetilde{\widetilde{h}}(\xi)=\left(K_{3}\right)^{q_{3} / q_{2}} h^{q_{3}}(\xi) R_{3}(\xi) \text {, } \\
& K_{1}=\left(\frac{\Gamma\left(1+p_{1}\left(\beta_{1}-1\right)\right)}{p_{1}^{1+p_{1}\left(\beta_{1}-1\right)}}\right)^{q_{1} / p_{1}}, \\
& K_{2}=\left(\frac{\Gamma\left(1+p_{2}\left(\beta_{2}-1\right)\right)}{p_{2}^{1+p_{2}\left(\beta_{2}-1\right)}}\right)^{q_{1} / p_{2}}, \\
& K_{3}=\left(\frac{\Gamma\left(1+p_{3}\left(\beta_{3}-1\right)\right)}{p_{3}^{1+p_{3}\left(\beta_{3}-1\right)}}\right)^{q_{2} / p_{3}}, \\
& T_{1}=\operatorname{Max}\left\{t \in I, \tilde{C}(t) \in \operatorname{Dom} \Omega_{3}^{-1}, \Omega_{3}^{-1}\{\tilde{C}(t)\} \in D o m \Omega_{2}^{-1},\right. \\
& \left.\Omega_{2}^{-1}\left\{\Omega_{3}^{-1}\{\tilde{C}(t)\}\right\} \in D o m \Omega_{1}^{-1}\right\} .
\end{aligned}
$$

Proof.

Let $p_{i}=1+\beta_{i}(i=1,2,3)$. Then

$$
\frac{1}{p_{i}}+\frac{1}{q_{i}}=\frac{1}{1+\beta_{i}}+\frac{\beta_{i}}{1+\beta_{i}}=1(i=1,2,3)
$$

and using the Holder inequality, we obtain from (3) that 


$$
\begin{aligned}
u(t) \leq & a(t)+\int_{t_{0}}^{t}(t-s)^{\beta_{1}-1} e^{s} f(s) e^{-s} w_{1}(u(s))[u(s) \\
& +\int_{t_{0}}^{s}(s-\tau)^{\beta_{2}-1} e^{\tau} g(\tau) e^{-\tau} w_{2}(u(\tau))[u(\tau) \\
& \left.\left.+\int_{t_{0}}^{\tau}(\tau-\xi)^{\beta_{3}-1} e^{\xi} h(\xi) e^{-\xi} w_{3}(u(\xi)) d \xi\right] d \tau\right] d s \\
\leq & a(t)+\left[\int_{t_{0}}^{t}(t-s)^{p_{1}\left(\beta_{1}-1\right)} e^{p_{1} s} d s\right]^{1 / p_{1}}\left[\int_{t_{0}}^{t} f^{q_{1}}(s) e^{-q_{1} s} w_{1}^{q_{1}}(u(s))[u(s)\right. \\
& +\left[\int_{t_{0}}^{s}(s-\tau)^{p_{2}\left(\beta_{2}-1\right)} e^{p_{2} \tau} d \tau\right]^{1 / p_{2}}\left[\int_{t_{0}}^{s} e^{-q_{2} \tau} g^{q_{2}}(\tau) w_{2}^{q_{2}}(u(\tau))[u(\tau)\right. \\
& +\left[\int_{t_{0}}^{\tau}(\tau-\xi)^{p_{3}\left(\beta_{3}-1\right)} e^{p_{3} \xi} d \xi\right]^{1 / p_{3}} \\
& \left.\left.\left.\left.\times\left[\int_{t_{0}}^{\tau} e^{-q_{3} \xi} h^{q_{3}}(\xi) w_{3}^{q_{3}}(u(\xi)) d \xi\right]^{1 / q_{3}}\right]^{q_{2}} d \tau\right]^{1 / q_{2}}\right]^{q_{1}} d s\right]^{1 / q_{1}}, \forall t \in I .
\end{aligned}
$$

Using (5) in Lemma 1 and (6) in Lemma 2 with $n=2, \alpha=q_{1}$, from the above inequality we obtain that

$$
\begin{aligned}
u(t) \leq & a(t)+\left[\int_{t_{0}}^{t}(t-s)^{p_{1}\left(\beta_{1}-1\right)} e^{p_{1} s} d s\right]^{1 / p_{1}}\left[\int _ { t _ { 0 } } ^ { t } f ^ { q _ { 1 } } ( s ) e ^ { - q _ { 1 } s } w _ { 1 } ^ { q _ { 1 } } ( u ( s ) ) \left[2^{q_{1}-1} u^{q_{1}}(s)\right.\right. \\
& +2^{q_{1}-1}\left[\int_{t_{0}}^{s}(s-\tau)^{p_{2}\left(\beta_{2}-1\right)} e^{p_{2} \tau} d \tau\right]^{q_{1} / p_{2}} \\
& \times\left[\int _ { t _ { 0 } } ^ { s } e ^ { - q _ { 2 } \tau } g ^ { q _ { 2 } } ( \tau ) w _ { 2 } ^ { q _ { 2 } } ( u ( \tau ) ) \left[2^{q_{2}-1} u^{q_{2}}(\tau)\right.\right. \\
& +2^{q_{2}-1}\left[\int_{t_{0}}^{\tau}(\tau-\xi)^{p_{3}\left(\beta_{3}-1\right)} e^{p_{3} \xi} d \xi\right]^{q_{2} / p_{3}} \\
& \left.\left.\left.\left.\times\left[\int_{t_{0}}^{\tau} e^{-q_{3} \xi} h^{q_{3}}(\xi) w_{3}^{q_{3}}(u(\xi)) d \xi\right]^{q_{2} / q_{3}}\right] d \tau\right]^{q_{1} / q_{2}}\right] d s\right]^{1 / q_{1}} \\
\leq & a(t)+2^{q_{1}-1} K_{1}^{1 / q_{1}} e^{t}\left[\int _ { t _ { 0 } } ^ { t } f ^ { q _ { 1 } } ( s ) e ^ { - q _ { 1 } s } w _ { 1 } ^ { q _ { 1 } } ( u ( s ) ) \left[u^{q_{1}}(s)\right.\right. \\
& +2^{q_{2}-1} K_{2} e^{q_{1} s}\left[\int _ { t _ { 0 } } ^ { s } e ^ { - q _ { 2 } \tau } g ^ { q _ { 2 } } ( \tau ) w _ { 2 } ^ { q _ { 2 } } ( u ( \tau ) ) \left[u^{q_{2}}(\tau)\right.\right. \\
+ & \left.\left.\left.\left.K_{3} e^{q_{2} \tau}\left[\int_{t_{0}}^{\tau} e^{-q_{3} \xi} h^{q_{3}}(\xi) w_{3}^{q_{3}}(u(\xi)) d \xi\right]^{q_{2} / q_{3}}\right] d \tau\right]^{q_{1} / q_{2}}\right] d s\right]^{1 / q_{1}}
\end{aligned}
$$

for all $t \in I$. Using the condition (4) in Definition 1 and the inequality (6) in Lemma 2 with $n=2, \alpha=q_{1}$, we obtain from (14) that

$$
\begin{aligned}
u^{q_{1}}(t) \leq & 2^{q_{1}-1} a^{q_{1}}(t)+2^{2 q_{1}-2} K_{1} e^{q_{1} t} \int_{t_{0}}^{t} f^{q_{1}}(s) R_{1}(s) e^{q_{1} s} w_{1}\left(e^{-q_{1} s} u^{q_{1}}(s)\right)\left[u^{q_{1}}(s) e^{-q_{1} s}\right. \\
& +2^{q_{2}-1} K_{2}\left[\int _ { t _ { 0 } } ^ { s } g ^ { q _ { 2 } } ( \tau ) R _ { 2 } ( \tau ) e ^ { q _ { 2 } \tau } w _ { 2 } ( e ^ { - q _ { 2 } \tau } u ^ { q _ { 2 } } ( \tau ) ) \left[u^{q_{2}}(\tau) e^{-q_{2} \tau}\right.\right.
\end{aligned}
$$




$$
\left.\left.\left.+K_{3}\left[\int_{t_{0}}^{\tau} h^{q_{3}}(\xi) R_{3}(\xi) w_{3}\left(e^{-q_{3} \xi} u^{q_{3}}(\xi)\right) d \xi\right]^{q_{2} / q_{3}}\right] d \tau\right]^{q_{1} / q_{2}}\right] d s, \forall t \in I .
$$

Let $v(t)=u^{q_{1}}(t) e^{-q_{1} t}$, we have from (15)

$$
\begin{aligned}
v(t) \leq B(t) & +\int_{t_{0}}^{t} \tilde{\widetilde{f}}(s) w_{1}(v(s))\left[v(s)+\int_{t_{0}}^{s} \widetilde{\widetilde{g}}(\tau) w_{2}(v(\tau))[v(\tau)\right. \\
& \left.\left.\left.+\left[\int_{t_{0}}^{\tau} \tilde{\widetilde{h}}(\xi) w_{3}(v(\xi)) d \xi\right]^{q_{2} / q_{3}}\right] d \tau\right]^{q_{1} / q_{2}}\right] d s, \forall t \in I,
\end{aligned}
$$

Let $Z_{1}(t)$ denote the function on the right-hand side of (16), which is a positive and nondecreasing function on $I$. From (16), we have

$$
v(t) \leq z_{1}(t), B(t) \leq z_{1}(t), \forall t \in I .
$$

Differentiating $z_{1}(t)$ with respect to $t$, using (17) we have

$$
\begin{aligned}
& z_{1}^{\prime}(t)=B^{\prime}(t)+\widetilde{\widetilde{f}}(t) w_{1}(v(t))\left[v(t)+\int_{t_{0}}^{t} \widetilde{\widetilde{g}}(\tau) w_{2}(v(\tau))[v(\tau)\right. \\
& \left.\left.\left.+\left[\int_{t_{0}}^{\tau} \tilde{h}(\xi) w_{3}(v(\xi)) d \xi\right]^{a_{2} / q_{3}}\right] d \tau\right]^{q_{1} / q_{2}}\right] \\
& \leq B^{\prime}(t)+\widetilde{\widetilde{f}}(t) w_{1}\left(z_{2}(t)\right) z_{2}(t), \forall t \in I,
\end{aligned}
$$

where

$$
\begin{aligned}
z_{2}(t)=z_{1}(t) & +\left[\int _ { t _ { 0 } } ^ { t } \tilde { g } ( \tau ) w _ { 2 } ( z _ { 1 } ( \tau ) ) \left[z_{1}(\tau)\right.\right. \\
& \left.\left.+\left[\int_{t_{0}}^{\tau} \tilde{\widetilde{h}}(\xi) w_{3}\left(z_{1}(\xi)\right) d \xi\right]^{q_{2} / q_{3}}\right] d \tau\right]^{q_{1} / q_{2}} .
\end{aligned}
$$

Obviously,

$$
z_{2}\left(t_{0}\right)=z_{1}\left(t_{0}\right), z_{1}(t) \leq z_{2}(t), \forall t \in I .
$$

since $q_{1}<q_{2}<q_{3}, q_{2} / q_{1}>1$. Using the inequality (6) in Lemma 2 with $n=2, \alpha=q_{2} / q_{1}$, we obtain that

$$
\begin{gathered}
z_{2}^{q_{2} / q_{1}}(t)=2^{\frac{q_{2}}{q_{1}}-1} z_{1}^{q_{2} / q_{1}}(t)+2^{\frac{q_{2}}{q_{1}}-1} \int_{t_{0}}^{t} \widetilde{\widetilde{g}}(\tau) w_{2}\left(z_{1}(\tau)\right)\left[z_{1}(\tau)\right. \\
\left.+\left[\int_{t_{0}}^{\tau} \widetilde{\tilde{h}}(\xi) w_{3}\left(z_{1}(\xi)\right) d \xi\right]^{q_{2} / q_{3}}\right] d \tau .
\end{gathered}
$$

From (18) and (20), we have

$$
\begin{aligned}
& \frac{q_{2}}{q_{1}} z_{2}^{\frac{q_{2}}{q_{1}}-1}(t) z_{2}^{\prime}(t)=\frac{q_{2}}{q_{1}} 2^{\frac{q_{2}}{q_{1}}-1} z_{1}^{\frac{q_{2}}{q_{1}}-1}(t) z_{1}^{\prime}(t)+2^{\frac{q_{2}}{q_{1}}-1} \tilde{\widetilde{g}}(t) w_{2}\left(z_{1}(t)\right)\left[z_{1}(t)\right. \\
& \left.+\left[\int_{t_{0}}^{t} \widetilde{\widetilde{h}}(\xi) w_{3}\left(z_{1}(\xi)\right) d \xi\right]^{q_{2} / q_{3}}\right]
\end{aligned}
$$




$$
\begin{gathered}
\leq \frac{q_{2}}{q_{1}} 2^{\frac{q_{2}}{q_{1}}-1} z_{2}^{\frac{q_{2}}{q_{1}}-1}(t)\left(B^{\prime}(t)+\tilde{\widetilde{f}}(t) w_{1}\left(z_{2}(t)\right) z_{2}(t)\right) \\
+2^{\frac{q_{2}}{q_{1}}-1} \widetilde{\widetilde{g}}(t) w_{2}\left(z_{2}(t)\right)\left[z_{2}(t)\right. \\
\left.\quad+\left[\int_{t_{0}}^{t} \widetilde{\widetilde{h}}(\xi) w_{3}\left(z_{1}(\xi)\right) d \xi\right]^{q_{2} / q_{3}}\right] \\
=\frac{q_{2}}{q_{1}} 2^{\frac{q_{2}}{q_{1}}-1} z_{2}^{\frac{q_{2}}{q_{1}}-1}(t)\left(B^{\prime}(t)+\widetilde{\widetilde{f}}(t) w_{1}\left(z_{2}(t)\right) z_{2}(t)\right) \\
+2^{\frac{q_{2}}{q_{1}}-1} \tilde{\widetilde{g}}(t) w_{2}\left(z_{2}(t)\right) z_{3}(t),
\end{gathered}
$$

for all $t \in I$, where $z_{3}(t)=z_{2}(t)+\left[\int_{t_{0}}^{t} \tilde{\widetilde{h}}(\xi) w_{3}\left(z_{2}(\xi)\right) d \xi\right]^{q_{2} / q_{3}}$. Obviously, $z_{2}(t) \leq z_{3}(t)$. Using the inequality (6) with $n=2, \alpha=q_{3} / q_{2}>1$, we obtain that

$$
z_{3}^{q_{3} / q_{2}}(t)=2^{\frac{q_{3}}{q_{2}}-1} z_{2}^{q_{3} / q_{2}}(t)+2^{\frac{q_{3}}{q_{2}}-1} \int_{t_{0}}^{t} \approx \widetilde{h}(\xi) w_{3}\left(z_{2}(\xi)\right) d \xi . \forall t \in I .
$$

From (21) and (22), we have

$$
\begin{aligned}
\frac{q_{3}}{q_{2}} z_{3}^{\frac{q_{3}}{q_{2}}-1}(t) z_{3}^{\prime}(t)= & \frac{q_{3}}{q_{2}} 2^{\frac{q_{3}}{q_{2}}-1} z_{2}^{\frac{q_{3}}{q_{2}}-1}(t) z_{2}^{\prime}(t)+2^{\frac{q_{3}}{q_{2}}-1} \widetilde{\widetilde{h}}(t) w_{3}\left(z_{2}(t)\right) \\
\leq & \frac{q_{3}}{q_{2}} 2^{\frac{q_{3}}{q_{2}}+\frac{q_{2}}{q_{1}}-2} z_{3}^{\frac{q_{3}}{q_{2}}-1}(t)\left(B^{\prime}(t)+\widetilde{\widetilde{f}}(t) w_{1}\left(z_{3}(t)\right) z_{3}(t)\right) \\
& +\frac{q_{1} q_{3}}{q_{2}^{2}} 2^{\frac{q_{3}}{q_{2}}+\frac{q_{2}}{q_{1}}-2} z_{3}^{\frac{q_{3}}{q_{2}}-\frac{q_{2}}{q_{1}}}(t) \tilde{\tilde{g}}(t) w_{2}\left(z_{3}(t)\right) z_{3}(t) \\
& +2^{\frac{q_{3}}{q_{2}}-1} \tilde{h}(t) w_{3}\left(z_{3}(t)\right),
\end{aligned}
$$

for all $t \in I$. From (23) we have

$$
\begin{aligned}
z_{3}^{\prime}(t) \leq & \left.2^{\frac{q_{3}}{q_{2}}+\frac{q_{2}}{q_{1}}-2} B^{\prime}(t)+2^{\frac{q_{3}}{q_{2}}+\frac{q_{2}}{q_{1}}-2} \tilde{\tilde{f}}(t) w_{1}\left(z_{3}(t)\right) z_{3}(t)\right) \\
& +\frac{q_{1}}{q_{2}} 2^{\frac{q_{3}}{q_{2}}+\frac{q_{2}}{q_{1}}-2} \tilde{\widetilde{g}}(t) w_{2}\left(z_{3}(t)\right) z_{3}^{2-\frac{q_{2}}{q_{1}}}(t)+2^{\frac{q_{3}}{q_{2}}-1} \tilde{\widetilde{h}}(t) w_{3}\left(z_{3}(t)\right)
\end{aligned}
$$




$$
\begin{aligned}
= & C^{\prime}(t)+\tilde{f}(t) w_{1}\left(z_{3}(t)\right) z_{3}(t)+\tilde{g}(t) w_{2}\left(z_{3}(t)\right) z_{3}{ }^{2-\frac{q_{2}}{q_{1}}}(t) \\
& +\tilde{h}(t) w_{3}\left(z_{3}(t)\right), \forall t \in I .
\end{aligned}
$$

From (24) we have

$$
\begin{aligned}
v(t) \leq & \Omega_{1}^{-1}\left\{\Omega _ { 4 } ^ { - 1 } \left\{\Omega _ { 5 } ^ { - 1 } \left\{\Omega _ { 5 } \left[\Omega _ { 4 } \left(\Omega_{1}(C(t))+\int_{t_{0}}^{t} \tilde{h}(s) d s\right.\right.\right.\right.\right. \\
& \left.\left.\left.\left.+\int_{t_{0}}^{t} \tilde{g}(s) d s\right]+\int_{t_{0}}^{t} \tilde{f}(s) d s\right\}\right\}\right\}, \forall t \in\left[t_{0}, T_{1}\right],
\end{aligned}
$$

In view of $v(t)=u^{q_{1}}(t) e^{-q_{1} t}$, we can obtain (7).

\section{Summary}

In this paper, we establish a class of new nonlinear weakly singular integral inequality, which consists of iterated integral, and weakly singular integral kernel be involved in each layer. Under several practical assumptions, the inequality is solved by adopting novel analysis techniques, such as: change of variable, amplification method, differential and integration, inverse function, and the dialectical relationship between constants and variables, and explicit bounds for the unknown functions are given clearly.

\section{Acknowledgements}

This research was supported by National Natural Science Foundation of China (Project No.11161018). The corresponding author is Yun Ouyang.

\section{References}

[1] M. Medved, A new approach to an analysis of Henry type integral inequalities and their Bihari type versions, J Math Anal Appl.vol. 214, pp. 349-366, 1997.

[2] A. Abdeldaim, M. Yakout. On some new integral inequalities of GronwallBellman-Pachpatte type. Appl Math Comput.vol. 217, pp. 7887-7899, 2011.

[3] M. Kuczma, An introduction to the theory of functional equations and inequalities: Cauchys equation and Jensens inequality, Katowice: University of Katowice, 1985.

[4] M. Medved, Nonlinear singular integral inequalities for functions in two and $\mathrm{n}$ independent variables, J Inequal Appl. vol. 5, no. 3, pp. 287-308, 2000.

[5] M. Medved, On singular versions of Bihari and Wendroff-Pachpatte type integral inequalities and their application, Tatra Mt Math Publ. vol. 38, pp. 163-174, 2007.

[6] Q. H. Ma, J. Pecaric, Some new explicit bounds for weakly singular integral inequalities with applications to fractional differential and integral equations, J Math Anal Appl.vol. 341, pp. 894-905, 2008. 
[7] H. Wang, K. Zheng, Some nonlinear weakly singular integral inequalities with two variables and applications, J Inequal Appl. vol. 2010, Article ID 345701, pp. 1-12, 2010.

[8] Q. H. Ma, E. H. Yang, Bounds on solutions to some nonlinear Volterra integral inequalities with weakly singular kernels, Ann Di_er Equ. vol. 27, no. 3, pp. 283-292, 2011.

[9] K. Zheng, Bounds on some new weakly singular Wendroff-type integral inequalities and applications, J Inequal Appl.vol. 2013:159, pp. 1-11, 2013. 